\title{
Centro de Atenção Psicossocial: Panorama das Publicações de 1997 a 2008
}

\author{
Psychosocial Care Center - \\ Publications from 1997 to 2008: An Overview \\ Centro de Atención Psicosocial: \\ Panorama de Las Publicaciones de 1997 a 2008
}

Maria Luisa Gazabim Simões Ballarin, Iara Monteiro Smeke de Miranda \& Ana Carolina de Ramos Castelhano Fuentes

Pontifíca Universidade Católica de Campinas

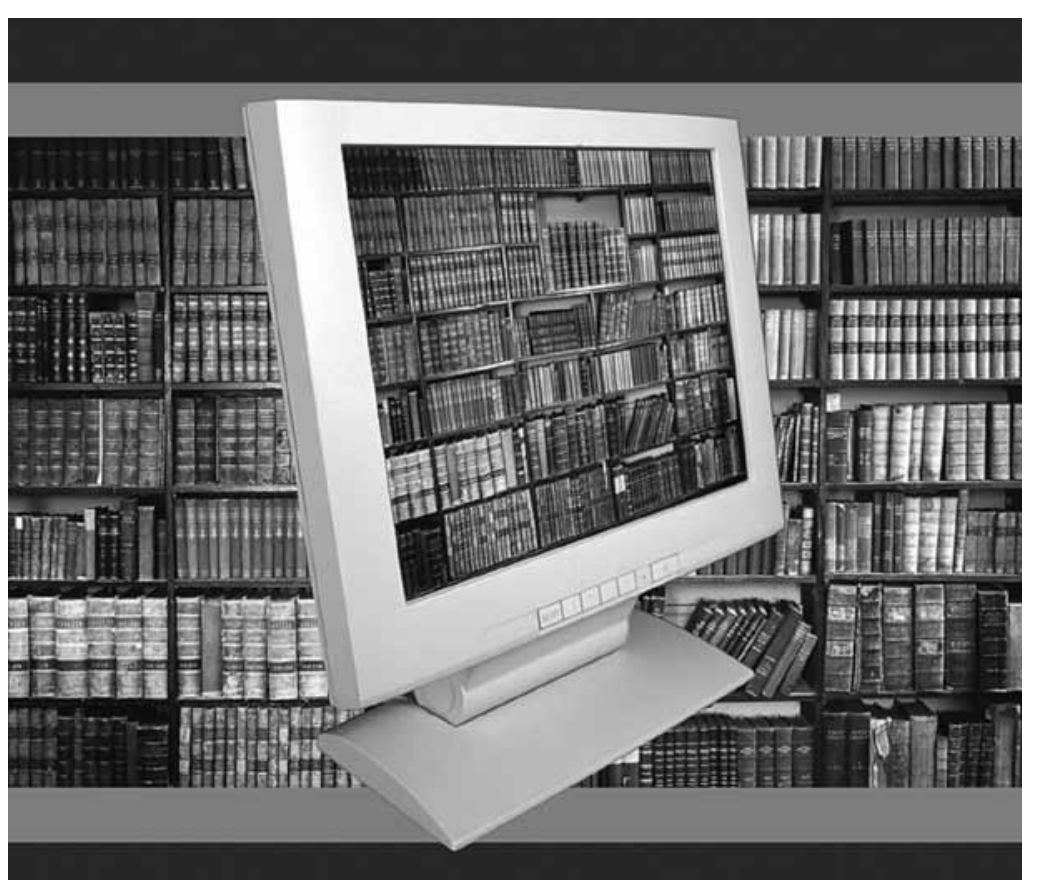


Resumo: Os Centros de Atenção Psicossocial (CAPS) se caracterizam como serviços estratégicos na articulação da rede de atenção à saúde mental e têm sido implantados gradualmente, a partir das transformações que ocorreram na assistência psiquiátrica brasileira, considerando-se o movimento de desinstitucionalização do doente mental, a reabilitação psicossocial e a reforma psiquiátrica. Por se tratar de serviços que determinam uma história recente de atenção à saúde mental, merecem ser sistematicamente estudados. Assim, este trabalho tem por objetivo analisar as publicações científicas relacionadas aos CAPS produzidas entre 1997 e 2008. Este estudo se caracteriza pela natureza descritiva, quantitativo-qualitativa, em que se realizou pesquisa bibliográfica com consulta às bases de dados LILACS e SciELO. Os resultados evidenciaram o predomínio de trabalhos publicados em periódicos científicos, sendo prevalentes as temáticas relacionadas aos serviços que compõem a rede de saúde mental, a organização, a avaliação e os projetos desenvolvidos nos CAPS, a análise teórica a respeito da reforma psiquiátrica e os estudos clínicos, socioantropológicos e epidemiológicos. A investigação demonstrou a relevância da identificação dos principais aspectos da produção científica relativa aos CAPS considerando-se o contexto da reforma psiquiátrica e as novas formas de atendimento em saúde mental, possibilitando a apreensão de uma visão panorâmica sobre esse campo.

Palavras-chave: Centro de Atenção Psicossocial (CAPS). Pesquisa bibliográfica. Reforma psiquiátrica. Serviços de saúde mental. Abstract: The Psychosocial Care Centers (CAPS) are characterized as strategic services in the articulation
of mental health care and attention network services and have been gradually introduced due to the
changes that occurred in the psychiatric care in Brazil, considering the deinstitutionalization of the
mentally ill people, the psychosocial rehabilitation and the psychiatric reform movements. Considered as
services that determine a recent history of attention to mental health, they deserve to be systematically
studied. This paper aims to examine the scientific publications related to the CAPS produced between
1997 and 2008. It is characterized as a descriptive and quantitative-qualitative study. The bibliographical
research was carried out using LILACS and SciELO databases. The results showed a predominance of
papers published in scientific journals, and the themes related to the services offered by the mental
health network, the organization, the evaluation and the projects developed at the CAPS, the theoretical
analyses of the psychiatric reform and the clinical, epidemiological and socio-anthropological studies
prevailed. The research showed the relevance of identifying the key aspects of the scientific production
on CAPS, considering the psychiatric reform context and the new ways of giving assistance in mental
health, thus allowing the apprehension of a panoramic view of this field of study.

Keywords: Psychosocial Care Center (CAPS). Bibliographical research. Psychiatric reform. Mental health services.

Resumen: Los Centros de Atención Psicosocial (CAPS) se caracterizan como servicios estratégicos en la articulación de la red de atención a la salud mental y han sido implantados gradualmente, a partir de las transformaciones que ocurrieron en la asistencia psiquiátrica brasileña, considerándose el movimiento de desinstitucionalización del enfermo mental, la rehabilitación psicosocial y la reforma psiquiátrica. Por tratarse de servicios que determinan una historia reciente de atención a la salud mental, merecen ser sistemáticamente estudiados. Así, este trabajo tiene por objetivo analizar las publicaciones científicas relacionadas a los CAPS producidas entre 1997 y 2008. Este estudio se caracteriza por la naturaleza descriptiva, cuantitativo-cualitativa, en que se realizó pesquisa bibliográfica con consulta a las bases de datos LILACS y SciELO. Los resultados evidenciaron el predominio de trabajos publicados en periódicos científicos, siendo prevalentes las temáticas relacionadas a los servicios que componen la red de salud mental, la organización, la evaluación y los proyectos desarrollados en los CAPS, el análisis teórico a respecto de la reforma psiquiátrica y los estudios clínicos, socio-antropológicos y epidemiológicos. La investigación demostró la relevancia de la identificación de los principales aspectos de la producción científica relativa a los CAPS considerando-se el contexto de la reforma psiquiátrica y las nuevas formas de atendimiento en salud mental, posibilitando la aprehensión de una visión panorámica sobre ese campo. Palabras clave: Centro de Atención Psicosocial (CAPS). Pesquisa bibliográfica. Reforma psiquiátrica. Servicios de salud mental. 
O objetivo deste trabalho foi realizar uma análise acerca das publicações científicas relacionadas aos Centros de Atenção Psicossocial (CAPS) produzidas entre os anos 1997 e 2008. O interesse em identificar as características dessa produção científica justifica-se na medida em que os CAPS assumem papel relevante como equipamentos estratégicos na estruturação da rede de cuidado em saúde mental. Atribui-se aos CAPS o direcionamento local das políticas e dos programas de saúde mental, através do desenvolvimento de projetos terapêuticos e comunitários, do encaminhamento e acompanhamento dos usuários dos Serviços Residenciais Terapêuticos e do assessoramento às equipes de Saúde da Família (Brasil, 2004). Além disso, tanto o Ministério da Saúde como alguns autores da reforma psiquiátrica apontam a necessidade de se ampliar o debate científico e a produção de conhecimento pertinente a temas relativos à assistência em saúde mental. Especialmente nesses seis últimos anos, o Ministério da Saúde centrou nos CAPS o maior investimento para expansão, consolidação e qualificação da rede de atenção à saúde mental no País, o que proporcionou um aumento de aproximadamente $102 \%$ no número de estabelecimentos no período (Brasil, 2007).

Caracterizados como serviços substitutivos ao hospital psiquiátrico tradicional, os CAPS atualmente podem ser classificados em I, II, III, ad (álcool e drogas) e i (infantil), conforme abrangência da população atendida e horário de funcionamento. Contemplam como objetivo fundamental o atendimento à crise em saúde mental e devem estar articulados à rede de serviços de saúde e a outras redes sociais de setores afins, para que se possa fazer frente à complexidade das demandas de inclusão (Brasil, 2004).

Embora, os CAPS e os Núcleos de Atenção Psicossocial (NAPS) tenham sido instituídos formalmente em 1992, através da Portaria/
SNAS n 224, do Ministério da Saúde, o estabelecimento e a consolidação desse tipo de serviço dependem de ações constituintes de um processo em construção, caracterizado pela implementação de uma nova forma de atendimento em saúde mental. É correto afirmar que especificamente nesta última década, inúmeras medidas foram tomadas no sentido de redirecionar o modelo assistencial em saúde mental. Nesse contexto, a promulgação da Lei no 10.216 , de 2001 (Brasil, 2001), que dispõe sobre a proteção e os direitos das pessoas portadoras de transtornos mentais, a criação de programas estratégicos, interdisciplinares e permanentes que promovem a qualificação da rede de atenção psicossocial bem como as Portarias no 336, de 19 de fevereiro de 2002, e n 189 , de 20 de março de 2002, e a publicação do manual intitulado Saúde Mental no SUS: os Centros de Atenção Psicossocial, em 2004 (Brasil, 2004), vêm consolidando os objetivos da reforma psiquiátrica como política de governo.

\section{Metodologia}

Este estudo possui natureza descritiva, quantitativo-qualitativa, em que se realizou levantamento sobre a produção científica relacionada aos CAPS. A pesquisa bibliográfica foi realizada por meio de busca eletrônica das produções científicas indexadas na base de dados da Biblioteca Virtual em Saúde (BIREME, http://www.bireme.br/), que, sendo um centro especializado da Organização Panamericana de Saúde (OPAS), abarca vasta produção científica. A busca de informações na BIREME direcionou o estudo para as bases de dados Literatura Latino-Americana e do Caribe em Ciências da Saúde (LILACS, http://bases.bireme.br/ cgi-bin/wxislind.exe/iah/cys/?lsisScript=iah/ iah. $x$ is\&base $=$ LILACS\&lang $=p$ ) e Scientific Electronic Library Online (SciELO, http://www. scielo.br/). Para tanto, foram utilizados os descritores: centro de atenção psicossocial, 
núcleo de atenção psicossocial, serviços substitutivos de saúde mental e serviços comunitários de saúde mental.

Faz-se necessário destacar que não foram consultadas outras bases de dados, como o Instituto Brasileiro de Informação em Ciência e Tecnologia (IBICT) ou o Index Psi Teses. Ambas forneceriam subsídios relevantes para o estudo em questão, já que divulgam informações de trabalhos acadêmicos (dissertações e teses) apresentados aos programas brasileiros de pós-graduação na área da saúde e da Psicologia, respectivamente, e possibilitariam ampliar a amostra e, em consequência, as análises. A não inclusão de outras bases de dados evidencia os limites deste estudo. Contudo, segundo Passos (2003), é "necessário reconhecer que todo banco de dados, por mais representativo que seja (...), possui contingências de indexação, o que pode determinar bias na pesquisa" (p. 232).

Considerou-se, portanto, que os descritores utilizados eram representativos das publicações relacionadas ao objetivo deste trabalho. As consultas cobriram os trabalhos publicados no período de 1997 a outubro de 2008, e o levantamento ocorreu entre os meses de agosto a outubro de 2008.

Os dados subsidiaram a elaboração de uma planilha que facilitou a sistematização das informações. Inicialmente, somente os resumos das publicações foram analisados. Seguindo-se a perspectiva de análise temática, histórica e processual, procedeu-se à leitura por amostragem na íntegra de alguns artigos. Em seguida, buscou-se identificar os eixos temáticos relacionados aos objetivos da investigação aferindo-se seus núcleos de sentido. Ressalta-se que a análise qualitativa foi complementada por consultas a livros e documentos oficiais não constantes das bases de dados consultadas. A esse respeito, Passos (2003) alerta sobre a necessidade de se levar em consideração a existência de uma grande e relevante produção nacional no campo da saúde mental que aborda experiências, projetos e reflexões teóricas não publicadas formalmente.

A amostra inicial encontrada sob os descritores utilizados foi de 398 publicações. Dessa amostra, excluindo-se as publicações que não correspondiam ao período estudado (142), aquelas que tratavam de títulos de publicações repetidos (63) e outras, cuja temática e objeto de estudo não estabeleciam relação com o tema estudado (33), chegou-se, portanto, à amostra final de 160 publicações.

\section{Resultados e discussão}

A análise dos dados coletados evidenciou que as publicações incluem tanto artigos publicados em periódicos científicos, como capítulos de livro, trabalhos acadêmicos - especialização, mestrado e doutorado -, e publicações de órgãos oficiais, de acordo com a Tabela 1, que compreendem trabalhos publicados em português, inglês e espanhol.

Tabela 1. Distribuição dos trabalhos por quadriênio e tipo de publicação.

\begin{tabular}{llcccc}
\hline Quadriênio & Qtd. & periódico & tese & oficial & capítulo do livro \\
\hline 1997 a 2000 & 35 & 28 & 4 & 3 & 0 \\
2001 a 2004 & 53 & 37 & 14 & 1 & 0 \\
2005 a 2008 & 72 & 64 & 5 & 1 & 1 \\
Total & 160 & 129 & 23 & 7 & 1 \\
\hline
\end{tabular}


Constata-se o predomínio de trabalhos publicados em periódicos científicos (129) em detrimento dos trabalhos acadêmicos (23) e de documentos oficiais (7), cuja forma de divulgação da pesquisa científica tem se mostrado relevante como ferramenta para disseminação do conhecimento científico. "A forma contemporânea predominante de comunicação e de disseminação científica são os artigos, tendo como meio as revistas acadêmicas que, por sua vez, compõem bases de dados nacionais, regionais e internacionais" (Minayo, 2007, p. 36). Segundo essa autora, a disseminação em progressão geométrica de artigos no cenário acadêmico brasileiro acompanha o aumento do volume dos cursos de pós-graduação strictu senso e a exigência de que os alunos publiquem suas pesquisas.

Faz-se necessário salientar que as bases de dados consultadas reúnem, em sua maioria, documentos publicados sob a forma de artigos científicos. No que se refere aos trabalhos acadêmicos, conforme já descrito, uma consulta ao IBICT (Instituto Brasileiro de Informação em Ciência e Tecnologia) e ao Index Psi Teses permitiria uma análise mais aprofundada. No entanto, questões de ordem técnica dificultaram essa consulta. Ainda assim, de acordo com Barreto e Barata (2008), a base LILACS é composta por aproximadamente $78 \%$ de artigos publicados em periódicos, seguida de 16\% de monografias, $5 \%$ de teses e o restante, de outros tipos de publicação. Os dados desta pesquisa coincidem com o estudo referido e reafirmam que os trabalhos publicados em periódicos são, de fato, a opção e a forma mais acessível de disponibilizar o conhecimento científico.

Foram identificados 56 diferentes periódicos com publicação de trabalhos relacionados aos termos indexadores utilizados neste estudo, compreendendo prioritariamente as áreas de enfermagem, saúde pública, psiquiatria, Psicologia, terapia ocupacional e Medicina (Tabela 2).

Tabela 2. Volume de publicações e periódicos, por área.

\begin{tabular}{lcl}
\hline Assunto & $\mathrm{N} 1$ & $\mathrm{~N} 2$ \\
\cline { 2 - 3 } do periódico & Artigos & Periódicos \\
\hline Enfermagem & 36 & 13 \\
Saúde pública & 31 & 12 \\
Psiquiatria & 26 & 9 \\
Psicologia & 16 & 12 \\
Terapia ocupacional & 8 & 1 \\
Medicina & 7 & 6 \\
Educação & 3 & 1 \\
Neurociências & 1 & 1 \\
Neurologia & 1 & 1 \\
Total & 129 & 56 \\
\hline
\end{tabular}

Do total de periódicos analisados, 10 deles publicaram 4 ou mais trabalhos, e os demais publicaram de 1 a 3 artigos (Tabela 3). Verificase que o Jornal Brasileiro de Psiquiatria publicou o maior número de artigos (11), seguido pela revista Texto \& Contexto Enfermagem (10), Revista de Terapia Ocupacional da USP (8), Cadernos de Saúde Pública (8), Revista LatinoAmericana de Enfermagem (Ribeirão Preto) (6), Revista da Escola de Enfermagem da USP (5), Ciência \& Saúde Coletiva (5), Revista Enfermagem - UERI (4), Psicologia: Ciência e Profissão (4), Cuadernos médico-sociales/ Colégio Médico de Chile (4), conforme a Tabela 3. 
Tabela 3. Quantidade de trabalhos publicados por periódico científico.

\begin{tabular}{ll}
\hline Periódico científico & $\mathrm{N}$ \\
\hline Jornal Brasileiro de Psiquiatria & 11 \\
Texto \& Contexto. Enfermagem & 10 \\
Revista de Terapia Ocupacional da Universidade de São Paulo & 8 \\
Cadernos de Saúde Pública (FIOCRUZ) & 8 \\
Revista Latino-Americana de Enfermagem & 6 \\
Revista da Escola de Enfermagem da USP & 5 \\
Ciência \& Saúde Coletiva & 5 \\
Revista Enfermagem (UERJ) & 4 \\
Psicologia: Ciência e Profissão & 4 \\
Cuademos médico-sociales/Colégio Médico de Chile & 4 \\
Outros* & 64 \\
Total & 129 \\
\hline
\end{tabular}

*periódicos com até 3 trabalhos publicados

Em relação à região de origem das publicações, destaca-se que a maioria corresponde à Região Sudeste do Brasil (60,6\%), seguidos da Região Sul (16,3\%), Centro-Oeste (6,3\%) e Nordeste $(2,5 \%)$. Não houve registro de publicações pertinentes à Região Norte. Dentre as publicações de trabalhos de outros países, que correspondem a aproximadamente $14 \%$ da amostra, o Chile foi o país com o número de publicações mais representativo (47\%), seguido dos Estados Unidos (22\%), Cuba, Jamaica e Uruguai (9\% cada) e Argentina (4\%).

Quanto ao conteúdo das publicações, considerando-se a análise qualitativa, constatou-se tratar de temáticas diversificadas que foram agrupadas e descritas a partir de categorias determinadas pelas investigadoras. Nesse sentido, há que se considerar a possibilidade de outros arranjos e classificações, ademais, o conteúdo das publicações analisadas evidencia perspectiva híbrida, ou seja, a temática de uma publicação/artigo caberia em mais de uma categoria. A Tabela 4 descreve a distribuição dos trabalhos, por temática, em ordem decrescente.

Tabela 4. Distribuição das publicações por temática

\begin{tabular}{llc}
\hline Categoria temática & N & Percentagem na amostra \\
\hline A. Rede de serviços de saúde mental & 93 & $58,1 \%$ \\
B. Organização dos CAPS e/ou NAPS & 24 & $15,0 \%$ \\
C. Estudos socioantropológicos & 19 & $11,9 \%$ \\
D. Análise teórica da reforma psiquiátrica & 9 & $5,6 \%$ \\
E. Trabalhador de serviços de saúde mental & 9 & $5,6 \%$ \\
F. Estudos epidemiológicos & 6 & $3,8 \%$ \\
Total & 160 & $100 \%$ \\
\hline
\end{tabular}

Os dados apresentados na Tabela 4 evidenciam a predominância de publicações que se relacionam às temáticas referentes à rede de serviços de saúde mental, quais sejam, estudos que abordam outros serviços de saúde mental, e não propriamente os CAPS. Esse achado decorre, 
em parte, da metodologia adotada para a coleta de informações nas bases de dados, que não se restringiu somente ao emprego dos descritores centro de atenção psicossocial e núcleo de atenção psicossocial.

Além disso, destaca-se o fato de que a constituição do tipo de serviço CAPS se caracteriza pela sua articulação com a rede de atenção à saúde estabelecida pelo SUS, o que, na visão das investigadoras, contribuiu para a inserção dos artigos na amostra de publicações sobre outros serviços de saúde que citam ou apenas fazem referência aos CAPS. Em seguida, encontram-se os textos sobre a organização do serviço $(15 \%)$, os estudos socioantropológicos $(11,9 \%)$, os de análise teórica sobre a reforma psiquiátrica $(5,6 \%)$, os relacionados ao trabalhador de serviços de saúde mental $(5,6 \%)$ e os estudos clínicos e epidemiológicos (3,8\%).

Especificamente em relação à categoria temática A, depreende-se que as publicações abordam, conforme já descrito, experiências relacionadas a outros serviços de saúde mental que compõem a rede de atenção em saúde mental, tais como Serviço Residencial Terapêutico (SRT), Enfermarias de Psiquiatria em Hospital Geral, cooperativas e ambulatórios de saúde mental, bem como ações de saúde mental na atenção básica e na Estratégia de Saúde da Família. Com relação aos SRT, embora os estudos de Mângia e Rosa (2002) e Vidal, Bandeira e Gontijo (2008) salientem a complexidade de que se reveste a organização e a implantação, na comunidade, desse tipo de serviço, bem como as dificuldades de articulação com os CAPS, os mesmos indicam que pacientes que viveram longos períodos de internação em muito podem se beneficiar com a inserção em moradias terapêuticas, na medida em que estas objetivam a sua reinserção social e o resgate de sua autonomia.

A inserção da assistência em saúde mental na atenção básica vem sendo descrita nos estudos de Feneric, Pereira e Zeoula (2004), Nunes, Jucá e Valentim (2007), Souza, Matias, Gomes e Parente (2007) e no de Vidal et al. (2008) como um processo ainda em implementação. Nesse sentido, as ações devem relacionar-se aos princípios de integralidade da atenção, participação social, ampliação do conceito saúdedoença, interdisciplinaridade do cuidado e territorialização, evidenciando a existência de princípios norteadores comuns entre a reforma psiquiátrica e a reforma sanitária.

A Estratégia de Saúde da Família seria também articuladora da rede de saúde mental, com vista à superação do modelo hospitalocêntrico e responsável por centrar o cuidado na família de modo a desenvolver ações de promoção e de prevenção de saúde mental, além de realizar práticas intersetoriais. Entretanto, Brêda, Rosa, Pereira e Scatena (2005) e Nunes et al. (2007) chamam a atenção para os impasses e as dificuldades existentes para a operacionalização efetiva dessa rede de cuidados em saúde mental. A falta de capacitação dos profissionais para manejar os casos de pacientes com transtornos mentais, o desconhecimento sobre a reforma psiquiátrica, a falta de recursos materiais e a falta de articulação com os próprios serviços de saúde mental que funcionam como retaguarda são apontados como fatores que dificultam a constituição de uma rede efetiva de cuidados. Além dos estudos descritos, ressalta-se que os de Mângia e Muramoto $(2005,2006)$ abordam diferentes aspectos da estruturação da rede de atenção e consideram questões culturais e estruturais, redes sociais e território.

Com relação à categoria temática $B$, cujas publicações abordam a organização, a implementação e a avaliação de programas 
relativos aos CAPS, os trabalhos de Goldberg (1994), Guljor (2003), Silva (2005), OnockoCampos e Furtado (2006) e Schrank e Olschowsky (2008) resgatam alguns aspectos históricos relativos à implantação do CAPS Luiz Cerqueira, em 1987, o primeiro do Estado de São Paulo, bem como à do NAPS inaugurado em 1989, na cidade de Santos. A análise desses primeiros serviços que se estruturaram a partir do paradigma da atenção psicossocial possibilitou a constatação de que, em ambos, foi possível construir intervenções diferenciadas do modelo assistencial tradicional (Guljor, 2003). Nesse sentido, ambos se tornaram referência de serviços comunitários e de base territorial, visando à superação do modelo centrado no hospital psiquiátrico.

As publicações evidenciaram também uma tendência que atribui aos CAPS um papel relevante no cenário das novas práticas em saúde mental, como o estudo de Ribeiro (2004), que relata a criação de um CAPS no interior de São Paulo com vistas à construção de um serviço de atendimento extra-hospitalar, de trabalho em equipe interdisciplinar com respeito às subjetividades e singularidades dos sujeitos envolvidos. Os trabalhos de Soares e Saeki (2006), Schrank e Olschowsky (2008), Mecca e Castro (2008) e Wetzel, Kantorski e Souza (2008) enfatizam a efetividade do CAPS na substituição da internação de longos períodos e na implantação de formas de tratamento que não isolem os usuários das famílias e da comunidade. Dá-se ênfase às formas de tratamento que priorizam o oferecimento heterogêneo de vínculos e de atividades, incluindo-se as artísticas, o trabalho coletivo da equipe, o envolvimento de diferentes atores e a construção de projetos terapêuticos singularizados possuidores de potência e capazes de resgatar o sujeito em seu protagonismo.
Enquanto dispositivo estratégico na reversão do modelo hospitalar, cabe aos CAPS aglutinar e organizar a rede local de saúde mental. No entanto, análises consideram que essa é a meta a ser alcançada, já que ainda são inúmeros os desafios (OnockoCampos \& Furtado, 2006). Nesse sentido, para que os CAPS funcionem efetivamente como novos serviços, é fundamental que haja um compromisso de se ofertar um atendimento que coloque em evidência as inter-relações existentes entre subjetividade, gestão dos processos de trabalho e clínica, capaz, portanto, de romper o atendimento tradicionalmente oferecido.

Constatou-se, portanto, nas publicações relacionadas tanto à temática $\mathrm{A}$ como à $\mathrm{B}$, que, ao dirigir a atenção para as experiências relacionadas a outros serviços de saúde mental que compõem a rede de atenção em saúde mental, como cooperativas, SRT, ações de saúde mental na atenção básica, Estratégia de Saúde da Família e aos próprios CAPS, há uma tendência em salientar os avanços conquistados sem, contudo, ignorar os desafios ainda existentes para a consolidação de uma rede de atenção e cuidados efetivamente articulada. A perspectiva e a necessidade de avaliação constante dos serviços substitutivos, bem como os questionamentos acerca das práticas efetivadas no contexto de cada um desses serviços, direcionam as publicações.

As publicações enquadradas na categoria temática C contemplam os estudos socioantropológicos. Os trabalhos preconizam a ótica e a percepção dos usuários e familiares envolvidos na utilização dos serviços oferecidos pelos CAPS. Observa-se o predomínio de pesquisas realizadas em campo e que se utilizam de entrevistas semiestruturadas, como nos trabalhos de Crivelatti, Durman e Hofstatter (2006), Soares e Saeki (2006), Surjus (2007) e Pegoraro e Caldana (2008). Nota-se, ainda, uma preocupação em realizar discussões teóricas que fundamentem a 
necessidade da pesquisa, com vistas à caracterização dos CAPS como serviços substitutivos ao modelo de atendimento manicomial. Schmid (2007) problematiza a clínica da reforma psiquiátrica e a destaca como uma nova forma de abordagem do sofrimento psíquico, denominando-a clínica de percurso, através do relato do percurso de um jovem atendido no serviço a partir do referencial psicanalítico. A autora destaca, concomitantemente, a importância da família e dos vínculos sociais na atenção ao doente mental, enquanto Pegoraro e Caldana (2008) investigam a sobrecarga experienciada pelo familiar responsável pelo cuidado de usuários e tecem uma reflexão teórica acerca da importância da família no contexto de tratamento proposto pelas atuais políticas públicas de saúde mental, apontando a necessidade de também se colocar o cuidador como alvo de atenção dos serviços de saúde mental. Soares e Saeki (2006) abordam a percepção dos usuários do serviço, que reflete conceitos marcados por práticas tradicionais organicistas que ainda influenciam as ações profissionais, juntamente à postura de acolhimento e escuta, além de práticas que têm enfocado a melhora da qualidade de vida e a autonomia, com vistas ao apoio do enfrentamento de dificuldades do cotidiano.

Desse modo, as publicações da categoria temática $C$ expressam a preocupação em se compreender e problematizar as percepções, as experiências e vivências de trabalhadores, familiares e usuários de CAPS, sendo a subjetividade e a intersubjetividade a base sob a qual são elaboradas as diversas interpretações das experiências e do mundo. Foi possível constatar, ainda, que os trabalhos apresentavam abordagens metodológicas relacionadas com a Antropologia, a Psicologia e a Sociologia, com evidência de textos que se utilizam das teorias das representações sociais, da psicanálise e da fenomenologiahermenêutica.
Os trabalhos classificados na categoria temática $D$ destacam estudos que fundamentalmente analisam, do ponto de vista teórico, o processo de reforma psiquiátrica no Brasil. Os estudos de Tenório (2002), Yasui (2006) e Bezerra Jr. (2007) evidenciam alguns acontecimentos relevantes que antecederam o movimento da reforma psiquiátrica e analisam o arcabouço conceitual das políticas públicas dirigidas à saúde mental. Os artigos destacam a importância da mudança no paradigma da atenção a fim de superar o modelo de assistência asilar. Yasui (2006), baseando-se em Amarante (2003), reafirma as quatro dimensões fundamentais do processo de reforma psiquiátrica, quais sejam: a dimensão epistemológica, relativa ao campo da produção de saberes, a dimensão técnico-assistencial, relacionada à necessidade de constituição de uma rede de serviços e cuidados, a dimensão jurídico-política, que envolve discussões e mudanças na legislação, e a dimensão sociocultural, que envolve a discussão sobre a necessidade de transformação do imaginário em relação à loucura e as suas formas de tratamento. Um dos desafios impostos à reforma psiquiátrica refere-se à exigência da formação de profissionais capazes de exercitarem sua capacidade de reflexão crítica (Bezerra Jr., 2007), aspecto esse indispensável para a superação das dificuldades inerentes ao trabalho e para a sustentação de uma prática de cuidado transformadora, permitindo a construção de uma nova atitude epistemológica e ética frente ao fenômeno da loucura.

Depreende-se, portanto, que as publicações pertinentes a essa categoria temática destacam a importância de se compreender não só os CAPS, mas também os serviços substitutivos que compõem a rede de atenção de saúde mental, tendo-se em vista a heterogeneidade de arcabouços conceituais que fundamentam as práticas em curso, o processo histórico e o contexto criado pela reforma psiquiátrica. 
Apesar dos enfrentamentos cotidianos, esse processo vem permitindo a inserção social dos usuários e uma nova forma de se cuidar do sofrimento psíquico.

Enquadradas na categoria temática $E$, identificam-se as publicações de Rocha (2005), Rabelo e Torres (2005), Garcia e Jorge (2006) e Nardi e Ramminger (2007), que discorrem sobre os trabalhadores de saúde mental (enfermeiros, auxiliares de enfermagem, psicólogos e terapeutas ocupacionais, entre outros), inseridos em serviços constituídos a partir da reforma psiquiátrica, como os CAPS, considerando suas vivências, seu bem-estar físico e psicológico, a heterogeneidade de experiências de cuidado e as vicissitudes do trabalho em equipe, bem como o cotidiano do trabalho, sua relação com os usuários e familiares e os aspectos relativos à organização e aos processos de trabalho. $\mathrm{O}$ oferecimento de um cuidado diferenciado e de qualidade implica novo saber e novo posicionamento por parte dos profissionais da equipe; implica, sobretudo, a integração desses novos saberes e, em consequência, uma proposta de trabalho interdisciplinar.

As publicações enquadradas na categoria temática $\mathrm{F}$ englobam estudos como os de Pelisoli e Moreira $(2005,2007)$ e de Souza (2007), que visam a traçar o perfil epidemiológico de usuários de serviços de saúde mental como o CAPS. Os estudos evidenciam a importância de se efetivar investigações sobre a prevalência dos transtornos, a faixa etária, a hipótese diagnóstica, o perfil sociodemográfico, os tipos de atividades terapêuticas e a adesão dos usuários ao tratamento, entre outros aspectos relacionados ao atendimento e à população assistida nesses serviços. Especificamente em relação aos CAPS que prestam assistência a uma população infanto-juvenil, Hoffmann, Santos e Mota (2008) apontam o número reduzido de estudos sobre a utilização e os aspectos epidemiológicos dessa população, destacando a necessidade de aprimoramento do sistema de informações e de registros sobre esses serviços. Reafirmam ainda a necessidade de implementação de estudos desse tipo, objetivando adequar e planejar de modo mais efetivo o atendimento ofertado.

\section{Considerações finais}

O objetivo desta pesquisa foi identificar o panorama das publicações científicas referentes aos Centros de Atenção Psicossocial a partir da identificação e do estudo de trabalhos que abarcaram os últimos dez anos, possibilitando, assim, uma atualização acerca da produção de conhecimento. A amostra selecionada se mostrou pertinente aos propósitos deste estudo, na medida em que seu conteúdo possibilitou, através da verificação e da problematização das questões que envolvem a constituição, a implementação, o funcionamento e a articulação do serviço, destacar as principais tendências expressas.

Como contribuições, o estudo possibilitou identificar o aumento gradual do número de publicações em periódicos considerandose o período investigado, bem como os principais veículos de publicação. As temáticas abordadas compreenderam aspectos bastante diversificados, já que trataram desde aspectos pertinentes à constituição e à organização dos CAPS e a seu papel na articulação da rede de serviços de saúde mental até a análise teórica da reforma psiquiátrica brasileira.

De modo geral, há que se enfatizar, nas publicações analisadas, uma tendência que se refere à reversão do modelo hospitalar, com ampliação significativa da rede de atenção extra-hospitalar de base comunitária e territorial. Avanços na consolidação e na efetivação da rede de serviços foram apontados, enfatizando-se a importância do cuidado diferenciado, das práticas grupais, do trabalho em equipe, da necessidade 
de avaliação constante e da inclusão das diferentes perspectivas e atores envolvidos no processo de sua construção sem, contudo, ignorar as limitações e os desafios ainda existentes para que essa rede seja efetivamente articulada. Por fim, diante da diversidade e da complexidade dos aspectos abordados nas publicações relacionadas aos CAPS, sugere-se que novas pesquisas sejam conduzidas, de maneira a aprofundar alguns dos aspectos apresentados neste estudo.

Maria Luisa Gazabim Simões Ballarin

Doutora em saúde mental pela Pontifícia Universidade Católica de Campinas, São Paulo - SP - Brasil.

lara Monteiro Smeke de Miranda

Graduanda em Terapia Ocupacional pela Pontíficia Universidade Católica de Campinas, São Paulo - SP - Brasil. E-mail: iaramsmeke@globo.com

\section{Ana Carolina de Ramos Castelhano Fuentes}

Graduanda em Terapia Ocupacional pela Pontíficia Universidade Católica de Campinas, São Paulo - SP - Brasil. E-mail: anacarolina_fuentes@yahoo.com.br

Amarante, P. A. (2003). A (clínica) e a reforma psiquiátrica. In P. Amarante (Ed.), Archivos de saúde mental e atenção psicossocial (pp. 45-66). Rio de Janeiro: Nau.

Barreto, M. L., \& Barata, R. B. (2008). Revistas de saúde pública e epidemiologia publicados no Brasil e em outros países de língua portuguesa. Emerging Themes in Epidemiology, 5(18). Recuperado em 25 de março de 2009, da ETE-Online: http://www.ete-online.com/content/5/1/18

Bezerra Júnior, B. (2007). Desafios da reforma psiquiátrica no Brasil. Physis: Revista de Saúde Coletiva, 17(2), 243-250.

Brasil. (2001). Lei no. 10.216, de 6 de abril de 2001. Dispõe sobre a proteção e os direitos das pessoas portadoras de transtornos mentais e redireciona o modelo assistencial em saúde mental. Diário Oficial da União, seção 1.

Brasil. (2004). Saúde mental no SUS: os Centros de Atenção Psicossocial. Brasília, DF: Ministério da Saúde, Secretaria de Atenção à Saúde, Departamento de Ações Programáticas Estratégicas.

Brasil. (2007). Saúde mental no SUS: acesso ao tratamento e mudança do modelo de atenção. Relatório de Gestão 20032006. Brasília, DF: Ministério da Saúde. Secretaria de Atenção à Saúde/DAPE.

Brêda, M. Z., Rosa, W. A. G., Pereira, M. A. O., \& Scatena, M. C. 
M. (2005). Duas estratégias e desafios comuns: a reabilitação psicossocial e a saúde da família. Revista Latino-Americana de Enfermagem, 13(3), 450-452.

Crivelatti, M. M. B., Durman, S., \& Hofstatter, L. M. (2006). Sofrimento psíquico na adolescência. Texto \& Contexto Enfermagem, 15(spe), 64-70.

Feneric, S. R. G., Pereira, W. A. B., \& Zeoula, F. M. (2004). Saúde mental e programa de saúde da família: percepções do agente comunitário de saúde. Psicologia Hospitalar 2(2). Recuperado em 25 de maio de 2009, da SciELO: http://pepsic.bvs-psi.org.br/scielo.php?script =sci arttext\&pid $=$ S1677-74092004000200011\&lng =pt\&nr $\mathrm{m}=\mathrm{iso}$

Goldberg, J. (1994). A clínica da psicose: um projeto na rede pública. Rio de Janeiro: Te Corá.

Guljor, A. P. F. (2003). Os Centros de Atenção Psicossocial: um estudo sobre a transformação do modelo assistencial em saúde mental. Dissertação de Mestrado, Escola Nacional de Saúde Pública, Rio de Janeiro, RJ.

Hoffmann, M. C. C. L., Santos, D. N., \& Mota, E. L. A. (2008). Caracterização dos usuários e dos serviços prestados por Centros de Atenção Psicossocial Infanto-Juvenil. Cadernos de Saúde Pública, 24(3), 633-642.

Mângia, E. F., \& Muramoto, M. T. (2005). O estudo de redes sociais: apontamentos teóricos e contribuições para o campo da saúde. Revista de Terapia Ocupacional da Universidade de São Paulo, 16(1), 22-30.

Mângia, E. F., \& Muramoto, M. T. (2006). Integralidade construção de novas profissionalidades no contexto dos serviços substitutivos de saúde mental. Revista de Terapia Ocupacional da Universidade de São Paulo, 17(3), 115-122.

Mângia, E. F., \& Rosa, C. A. (2002). Desinstitucionalização e serviços residenciais terapêuticos. Revista de Terapia Ocupacional da Universidade de São Paulo, 13(2), 71-77.

Mecca, R. C., \& Castro, E. D. (2008). Experiência estética e cotidiano institucional: novos mapas para subjetivar espaços destinados à saúde mental. Interface - Comunicação, Saúde, Educação, 12(25), 377-386.

Minayo, M. C. S. (2007). A complexa dinâmica da divulgação científica: o caso da Revista Ciência \& Saúde Coletiva. Revista Eletrônica de Comunicação, Informação e Inovação em Saúde, 1(1), 35-44. Recuperado em 03 de junho de 2009, da RECIIS: http://www.reciis.cict.fiocruz.br/index. php/reciis/article/viewFile/48/53-

Nunes, M., Jucá, V. J., \& Valentim, C. P. B. (2007). Ações de saúde mental no Programa Saúde da Família: confluências e dissonâncias das práticas com os princípios das reformas psiquiátrica e sanitária. Cadernos de Saúde Pública, 23(10) 2375-2384.

Onocko-Campos, R. T., \& Furtado, J. P. (2006). Entre a saúde coletiva e a saúde mental: um instrumental metodológico para avaliação da rede de Centros de Atenção Psicossocial (CAPS) do Sistema Único de Saúde. Cadernos de Saúde Pública, 22(5), 1053-1062.

Passos, I. C. F. (2003). Cartografia da publicação brasileira em saúde mental: 1980-1996. Psicologia: Teoria e Pesquisa, 19(3), 231-239.

Pegoraro, R. F., \& Caldana, R. H. L. (2008). Sofrimento psíquico em familiares de usuários de um Centro de Atenção Psicossocial (CAPS). Interface - Comunicação, Saúde, Educação, 12(25), 295-307.
Pelisoli, C. L., \& Moreira, A. K. (2005). Caracterização epidemiológica dos usuários do Centro de Atenção Psicossocial Casa Aberta. Revista de Psiquiatria do Rio Grande do Sul, 27(3), 270-277.

Pelisoli, C. L., \& Moreira, A. K. (2007). Avaliação de um Centro de Atenção Psicossocial por meio do perfil de seus usuários. Mental, 5(8), 61-75.

Rabelo, I. V. M., \& Torres, A. R. R. (2005). Trabalhadores em saúde mental: relações entre práticas profissionais e bem-estar físico e psicológico. Psicologia: Ciência e Profissão, 25(4), 614-625.

Ribeiro, S. L. (2004). A criação do Centro de Atenção Psicossocial Espaço Vivo. Psicologia: Ciência e Profissão, 24(3), 92-99.

Rocha, R. M. (2005). O enfermeiro na equipe interdisciplinar do Centro de Atenção Psicossocial e a as possibilidades de cuidar. Texto \& Contexto Enfermagem, 14(3), 350-357.

Schmid, P. C. (2007). Viajando não, só sonhando! Revista do Departamento de Psicologia da Universidade Federal Fluminense, 19(1), 187-197. Recuperado em 03 de junho de 2009, da SciELO: http://www.scielo.br/scielo.php?script=sci arttext\&pid $=$ S0104-80232007000100014\&Ing $=$ en\&nr $\mathrm{m}=$ iso

Schrank, G., \& Olschowsky, A. (2008). O Centro de Atenção Psicossocial e as estratégias para inserção da família. Revista da Escola de Enfermagem da Universidade de São Paulo, 42(1), 127-134

Silva, M. B. B. (2005). Atenção psicossocial e gestão de populações: sobre os discursos e as práticas em torno da responsabilidade no campo da saúde mental. Physis: Revista de Saúde Coletiva, 15(1), 127-150.

Soares, S. R. R., \& Saeki, T. (2006). The Psychosocial Care Center on the users' point of view. Revista Latino-Americana de Enfermagem, 14(6), 923-929.

Souza, A. J. F., Matias, G. N., Gomes, K. F. A., \& Parente, A. C. M. (2007). A saúde mental no Programa de Saúde da Família. Revista Brasileira de Enfermagem, 60(4), 391-395.

Souza, A. R. (2007). Centro de Atenção Psicossocial: perfil epidemiológico dos usuários. Dissertação de Mestrado, Faculdade de Farmácia, Odontologia e Enfermagem, Universidade Federal do Ceará, Fortaleza, CE.

Surjus, L. T. L. S. (2007). Narrativas políticas: o olhar dos usuários sobre o CAPS (Centro de Atenção Psicossocial). Dissertação de Mestrado, Faculdade de Ciências Médicas, Universidade Estadual de Campinas, Campinas, SP.

Tenório, F. (2002). A reforma psiquiátrica brasileira, da década de 1980 aos dias atuais: história e conceito. História, Ciências, Saúde-Manguinhos, 9(1), 25-59.

Vidal, C. E. L., Bandeira, M., \& Gontijo, E. D. (2008). Reforma psiquiátrica e serviços residenciais terapêuticos. Jornal Brasileiro de Psiquiatria, 57(1), 70-79.

Wetzel, C., Kantorski, L. P., \& Souza, J. (2008). Centro de Atenção Psicossocial: trajetória, organização e funcionamento. Revista Enfermagem - UERJ, 16(1), 39-45.

Yasui, S. (2006). Rupturas e encontros: desafios da reforma psiquiátrica brasileira. Tese de Doutorado, Escola Nacional de Saúde Pública Sergio Arouca, Rio de Janeiro, RJ. 